OPEN

SUBJECT AREAS:

QUANTUM INFORMATION

QUANTUM MECHANICS

QUBITS

THEORETICAL PHYSICS

Received

9 July 2013

Accepted

7 August 2013

Published

22 August 2013

Correspondence and requests for materials should be addressed to J.-L.C. (cqtchenj@nus. edu.sg)

\section{Testing Leggett's Inequality Using Aharonov-Casher Effect}

\author{
Hong-Yi Su', Jing-Ling Chen ${ }^{1,2}$, Chunfeng Wu ${ }^{2,3}$, Dong-Ling Deng ${ }^{4} \&$ C. H. Oh ${ }^{2,5}$
}

\begin{abstract}
'Theoretical Physics Division, Chern Institute of Mathematics, Nankai University, Tianjin 300071, People's Republic of China, ${ }^{2}$ Centre for Quantum Technologies, National University of Singapore, 3 Science Drive 2, Singapore 1 17543, ${ }^{3}$ Pillar of Engineering Product Development, Singapore University of Technology and Design, 20 Dover Drive, Singapore 138682, ${ }^{4}$ Department of Physics and Michigan Center for Theoretical Physics, University of Michigan, Ann Arbor, Michigan 48109, USA, ${ }^{5}$ Department of Physics, National University of Singapore, 2 Science Drive 3, Singapore 117542.
\end{abstract}

Bell's inequality is established based on local realism. The violation of Bell's inequality by quantum mechanics implies either locality or realism or both are untenable. Leggett's inequality is derived based on nonlocal realism. The violation of Leggett's inequality implies that quantum mechanics is neither local realistic nor nonlocal realistic. The incompatibility of nonlocal realism and quantum mechanics has been currently confirmed by photon experiments. In our work, we propose to test Leggett's inequality using the Aharonov-Casher effect. In our scheme, four entangled particles emitted from two sources manifest a two-qubit-typed correlation that may result in the violation of the Leggett inequality, while satisfying the no-signaling condition for spacelike separation. Our scheme is tolerant to some local inaccuracies due to the topological nature of the Aharonov-Casher phase. The experimental implementation of our scheme can be possibly realized by a calcium atomic polarization interferometer experiment.

ell's inequality ${ }^{1,2}$ imposes bounds on correlations of different parties of multipartite systems based on local realism. However, the violation of Bell's inequality by quantum mechanics implies either locality or realism or both are untenable. In the debate of incompatibility between quantum mechanics and any local realistic hidden variable theory, experiments ${ }^{3-9}$ have supported quantum mechanics. Although for some time there existed loopholes of locality ${ }^{4,5,7,8}$ and detection ${ }^{6,9}$, they have been almost closed. The invalidity of local realism is a reasonably established fact. In 2003, Leggett ${ }^{10}$ derived a class of inequalities based on nonlocal realism. He assumed that the state of a subsystem has been predetermined by some variable $\lambda$ even before the measurement, and that the joint probabilities consist of a mixture of correlations that cannot be separable. Since only states of subsystems have been predetermined, the whole system may be nonlocal. After the pioneer work, the incompatibility of nonlocal realism and quantum mechanics was experimentally confirmed ${ }^{11-14}$. It was shown that quantum mechanics is neither local realistic nor nonlocal realistic.

Topological property of physical systems has given rise to many applications ranging from quantum field theory to quantum information science. An example is the Aharonov-Bohm (AB) effect ${ }^{15}$, in which a moving charge has its phase shifted in the presence of a confined magnetic field, though apparently it feels no net force. In 1984, Aharonov and Casher ${ }^{16}$ predicted a dual of the AB effect. In the Aharonov-Casher (AC) effect, the role of charge and magnetic flux is exchanged, i.e., when a neutral particle with magnetic moment moves around an impenetrable line charge, it also acquires some phase shifts. The AC effect is traditionally understood as a nonlocal and topological effect in which a particle with magnetic moment acquires shifted phase when moving in a topologically nontrivial region. In 1998 a scheme involving the AC effect to test local realism was proposed by Pati $^{17}$, and the violation of Bell's inequality indicates the nonlocality of the four-particle entangled state.

In this work, we advance the study of nonlocality of the AC effect and present a scheme to test Leggett's inequality by resorting to the AC effect. Due to the topological nature of the AC effect, our scheme is robust against some local inaccuracies. We shall test the two-qubit Leggett inequality in a physical system consisting of four neutral particles with magnetic moments, whose initial state is a product of the singlet state of pair $(1,2)$ and the triplet state of pair $(3,4)$. Pseudo-Pauli matrices are introduced such that one may view an entangled spin pair like pair $(1,4)$ or pair $(2,3)$ as a "single qubit", and hence four particles as a total "two-qubit" system. The influence of the AC effect on an entangled spin pair is found to be equivalent to a rotation in terms of the pseudoPauli operators. Moreover, based on the final state of the four particles, we focus on some specific joint probabilities satisfying the no-signaling condition and obtain a two-qubit-type correlation function that may violate 
the Leggett inequality. We also present some discussion on the implementation of our scheme in a calcium atomic polarization interferometer experiment.

\section{Results}

Testing leggett's inequality using the AC effect. In a nonlocal hidden variable model, one assumes that the joint probability for a bipartite system consists of statistical mixture of simpler correlations:

$$
P(\alpha, \beta \mid \vec{a}, \vec{b})=\int d \lambda \rho(\lambda) P_{\lambda}(\alpha, \beta \mid \vec{a}, \vec{b})
$$

where $\lambda$ is a set of hidden variables determining the system, $\rho(\lambda)$ distribution of $\lambda$; $\alpha, \beta$ are measurement outcomes, and $\vec{a}, \vec{b}$ measurement settings for two subsystems, respectively. An extra requirement is that $P_{\lambda}$ satisfies the no-signaling condition, i.e., $\sum_{\beta} P_{\lambda}(\alpha, \beta \mid \vec{a}, \vec{b})=\sum_{\beta} P_{\lambda}\left(\alpha, \beta \mid \vec{a}, \vec{b}^{\prime}\right)$ and $\sum_{\alpha} P_{\lambda}(\alpha, \beta \mid \vec{a}, \vec{b})=$ $\sum_{\alpha} P_{\lambda}\left(\alpha, \beta \mid \vec{a}^{\prime}, \vec{b}\right)$. Follow Branciard et al.'s derivation of the Leggett inequality ${ }^{14}$, one can define the correlations for a two-qubit system as $P_{\lambda}(\alpha, \beta \mid \vec{a}, \vec{b})=\frac{1}{4}\left(1+\alpha M_{\lambda}^{A}(\vec{a}, \vec{b})+\beta M_{\lambda}^{B}(\vec{a}, \vec{b})+\alpha \beta C_{\lambda}(\vec{a}, \vec{b})\right)$. Here $M_{\lambda}^{A}(\vec{a}, \vec{b})=\sum_{\alpha, \beta} \alpha P_{\lambda}(\alpha, \beta \mid \vec{a}, \vec{b}), \quad M_{\lambda}^{B}(\vec{a}, \vec{b})=\sum_{\alpha, \beta} \beta P_{\lambda}(\alpha, \beta \mid \vec{a}, \vec{b})$, $C_{\lambda}(\vec{a}, \vec{b})=\sum_{\alpha, \beta} \alpha \beta P_{\lambda}(\alpha, \beta \mid \vec{a}, \vec{b})$, and $\alpha, \beta= \pm 1 . M_{\lambda}^{A}$ and $M^{B}$ are expectation values (or marginals) at each respective measuring location. According to no-signaling condition, the marginals $M_{\lambda}^{A}$ and $M_{\lambda}^{B}$ can be locally described by their respective choices of measurement, i.e., $M_{\lambda}^{A}(\vec{a}, \vec{b})=M_{\lambda}^{A}(\vec{a})$ and $M_{\lambda}^{B}(\vec{a}, \vec{b})=M_{\lambda}^{B}(\vec{b})$. Leggett assumed that each subsystem can be described by a pure quantum state, then for the two-qubit case, each hidden variable determines a product state $\lambda \rightarrow|\vec{u}\rangle \otimes|\vec{v}\rangle$, where $\vec{u}, \vec{v}$ are vectors on the Poincaré sphere. We have $M_{\lambda}^{A}(\vec{a})=\langle\vec{\sigma} \cdot \vec{a}\rangle_{\lambda}=\vec{u}_{\lambda} \cdot \vec{a}, M_{\lambda}^{B}(\vec{b})=$ $\langle\vec{\sigma} \cdot \vec{b}\rangle_{\lambda}=\vec{v}_{\lambda} \cdot \vec{b}$, where $\vec{\sigma}=\left(\sigma^{x}, \sigma^{y}, \sigma^{z}\right)$ is the usual Pauli matrix vector. Since no further assumption of the bipartite correlation function is made, generally speaking, $C_{\lambda}(\vec{a}, \vec{b}) \neq M_{\lambda}^{A}(\vec{a}) M_{\lambda}^{B}(\vec{b})$. The Leggett inequality is of the following form ${ }^{14}$

$$
\left|C(\vec{a}, \vec{b}) \pm C\left(\vec{a}, \overrightarrow{b^{\prime}}\right)\right| \leq 2-\int d \lambda \rho(\lambda)\left|M_{\lambda}^{B}(\vec{b}) \mp M_{\lambda}^{B}\left(\overline{b^{\prime}}\right)\right|,
$$

where $\vec{a}$ describes the measurement setting for Alice, $\vec{b}, \overrightarrow{b^{\prime}}$ are the two measurement settings for Bob. Consider three settings $\vec{a}_{i}(i=1,2,3)$ for Alice and six settings $\vec{b}_{i}, \overrightarrow{b_{i}^{\prime}}(i=1,2,3)$ for Bob as given in Ref. 14, where $\vec{b}_{i}-\vec{b}_{i}=2 \sin \left(\varphi / 2 \vec{e}_{i}\right)$ with $\vec{e}_{i}(i=1,2,3)$ being an orthogonal basis, then one arrives at the Leggett inequality as

$$
\frac{1}{3} \sum_{i=1}^{3}\left(\left|C\left(\vec{a}_{i}, \vec{b}_{i}\right)+C\left(\vec{a}_{i}, \vec{b}_{i}{ }_{i}\right)\right|\right) \leq 2-\frac{2}{3}\left|\sin \frac{\varphi}{2}\right| .
$$

For the singlet state of two qubits, the quantum correlation function reads $C(\vec{a}, \vec{b})=\int d \lambda \rho(\lambda) C_{\lambda}(\vec{a}, \vec{b})=\langle\vec{\sigma} \cdot \vec{a} \otimes \vec{\sigma} \cdot \vec{b}\rangle=-\vec{a} \cdot \vec{b}$. Then the Leggett inequality reduces to a simpler form:

$$
2\left|\cos \frac{\varphi}{2}\right|+\frac{2}{3}\left|\sin \frac{\varphi}{2}\right| \leq 2 \text {. }
$$

Let us consider a system of four neutral spin-1/2 particles with magnetic moments in the presence of a line charge. In the AC configuration, the particles are moving in $x y$-plane and the line charge oriented along the third axis (the $z$-axis). The motion of the particles is influenced by the electric field of line charge. Each particle will acquire a phase when moving along the plane,

$$
\begin{aligned}
& |\uparrow\rangle_{j} \rightarrow e^{i \int_{\ell_{j}}\left(\vec{E} \times \vec{\mu}_{j}\right) \cdot d \vec{r}}|\uparrow\rangle_{j}=e^{i \frac{\varphi_{j}}{2}}|\uparrow\rangle_{j}, \\
& |\downarrow\rangle_{j} \rightarrow e^{-i \int_{\ell_{j}}\left(\vec{E} \times \vec{\mu}_{j}\right) \cdot d \vec{r}}|\downarrow\rangle_{j}=e^{-i \frac{\varphi_{j}}{2}}|\downarrow\rangle_{j},
\end{aligned}
$$

where $|\uparrow\rangle_{j},|\downarrow\rangle_{j}$ describe quantum states with spin up and spin down for the $j$-th particle, $\vec{E}$ is electric field intensity, $\varphi_{j}=\int_{\ell_{j}}\left(\vec{E} \times \vec{\mu}_{j}\right) \cdot d \vec{r}$ is the measurable phase accumulated during the evolution, and $\vec{\mu}_{j}$ is the magnetic moment for the $j$-th particle.

Denote the state for two spin-1/2 particles by $|S, M\rangle$ with total spin $S$ and magnetic quantum number $M$, then the singlet state and the triplet state with $M=0$ are given by

$$
\begin{aligned}
& |0,0\rangle=(|\uparrow \downarrow\rangle-|\downarrow \uparrow\rangle) / \sqrt{2}, \\
& |1,0\rangle=(|\uparrow \downarrow\rangle+|\downarrow \uparrow\rangle) / \sqrt{2},
\end{aligned}
$$

Under the transformation (5), the initial singlet state $|0,0\rangle_{m n}$ and triplet state $|1,0\rangle_{m n}$ of particles $m$ and $n$ become

$$
\begin{aligned}
|0,0\rangle_{m n} & \rightarrow \cos \frac{\varphi_{m}-\varphi_{n}}{2}|0,0\rangle_{m n}+i \sin \frac{\varphi_{m}-\varphi_{n}}{2}|1,0\rangle_{m n}, \\
|1,0\rangle_{m n} & \rightarrow i \sin \frac{\varphi_{m}-\varphi_{n}}{2}|0,0\rangle_{m n}+\cos \frac{\varphi_{m}-\varphi_{n}}{2}|1,0\rangle_{m n},
\end{aligned}
$$

namely, the states $|0,0\rangle_{m n}$ and $|1,0\rangle_{m n}$ evolve to the quantum states that are linear superpositions of themselves. This is a very notable feature of the AC effect influencing an entangled spin pair ${ }^{17,18}$. Equation (7) implies that $\{|0,0\rangle,|1,0\rangle\}$ may span a subspace, and in turn one may treat the spin pair as a "single qubit". To make this point explicit, let us abbreviate

$$
|\overline{0}\rangle \equiv|0,0\rangle, \quad|\overline{1}\rangle \equiv|1,0\rangle,
$$

then Eq. (7) can be recast as

$$
\left(\begin{array}{l}
|\overline{0}\rangle_{m n} \\
|\overline{1}\rangle_{m n}
\end{array}\right) \rightarrow\left(\begin{array}{cc}
\cos \frac{\varphi_{m}-\varphi_{n}}{2} & i \sin \frac{\varphi_{m}-\varphi_{n}}{2} \\
i \sin \frac{\varphi_{m}-\varphi_{n}}{2} & \cos \frac{\varphi_{m}-\varphi_{n}}{2}
\end{array}\right)\left(\begin{array}{l}
|\overline{0}\rangle_{m n} \\
|\overline{1}\rangle_{m n}
\end{array}\right) .
$$

Moreover, one defines the following pseudo-Pauli matrices as $\sum^{x}=|\overline{0}\rangle\langle\overline{1}|+| \overline{1}\rangle\left\langle\overline{0}\left|, \quad \sum^{y}=-i(|\overline{0}\rangle\langle\overline{1}|-| \overline{1}\rangle\langle\overline{0}|), \quad \sum^{z}=\right| \overline{0}\right\rangle\langle\overline{0}|-$ $|\overline{1}\rangle\langle\overline{1}|$, which share similar properties as the usual Pauli matrices, then Eq. (9) is nothing but a rotation

$$
\mathcal{R}_{m n}^{x}\left(\varphi_{m}-\varphi_{n}\right)=e^{i\left(\varphi_{m}-\varphi_{n}\right) \sum_{m n}^{x} / 2}
$$

along $x$-axis on the basis $\{|\overline{0}\rangle,|\overline{1}\rangle\}$ of the "single qubit".

Our scheme for testing the Leggett inequality by experiment involves two pairs of entangled spin-1/2 particles. Similar to Refs. 17,18 , we prepare the four particles entangled in two pairs $(1,2)$ and $(3,4)$ initially, and finally perform some proper projective measurements on particle pairs $(1,4)$ and $(2,3)$ to obtain the correlation function. Assume initially that particles 1 and 2 are emitted from a source $O_{12}$ with total spin $S_{12}=0$ and magnetic moment $M_{12}=0$; similarly, particles 3 and 4 are emitted from a source $O_{34}$ with $S_{34}=1$ and $M_{34}=0$. Namely, the initial state reads $\left|\Psi_{i}\right\rangle=|0,0\rangle_{12} \otimes$ $|1,0\rangle_{34}=\frac{1}{2}(|\uparrow \downarrow \downarrow \uparrow\rangle-|\downarrow \uparrow \uparrow \downarrow\rangle+|\uparrow \uparrow \downarrow \downarrow\rangle-|\downarrow \downarrow \uparrow \uparrow\rangle)_{1423}$, in the last step of which we have rearranged the particles in the order of "1423". Actually, $\left|\Psi_{\mathrm{i}}\right\rangle$ can be rewritten as

$\left|\Psi_{i}\right\rangle=\frac{1}{2}\left(|\overline{0}\rangle_{14}|\overline{1}\rangle_{23}-|\overline{1}\rangle_{14}|\overline{0}\rangle_{23}\right)+\frac{1}{2}(|\uparrow \uparrow \downarrow \downarrow\rangle-|\downarrow \downarrow \uparrow \uparrow\rangle)_{1423}$.

However, the last two terms of Eq. (11) will vanish when they are acted by any operator defined in the subspace $\overline{\mathcal{H}}=\left\{|\overline{0}\rangle_{14},|\overline{1}\rangle_{14}\right\}$ $\otimes\left\{|\overline{0}\rangle_{23},|\overline{1}\rangle_{23}\right\}$ Here we retain them for normalization. In fact, the initial state can be understood as a "singlet state" $\left|\Psi_{i}\right\rangle \propto \frac{1}{\sqrt{2}}$ $\left(|\overline{0}\rangle_{14}|\overline{1}\rangle_{23}-|\overline{1}\rangle_{14}|\overline{0}\rangle_{23}\right)$ of "two-qubit" without any confusion. 
Our experiment proposal is demonstrated in Fig. 1. The distance from $A$ to $B$ is supposed to be large enough so that the measurement of particle pair $(1,4)$ and that of particle pair $(2,3)$ are space-like, and thus no-signaling condition is satisfied. Due to Eqs. (9) and (10), we have the final state of the four particles as

$$
\begin{aligned}
\left|\Psi_{\mathrm{f}}\right\rangle= & \frac{1}{2} \mathcal{R}_{A}^{x}\left(\varphi_{A}\right) \otimes \mathcal{R}_{B}^{x}\left(\varphi_{B}\right)\left(|\overline{0}\rangle_{14}|\overline{1}\rangle_{23}-|\overline{1}\rangle_{14}|\overline{0}\rangle_{23}\right)+ \\
& \frac{1}{2}\left(e^{i \gamma}|\uparrow \uparrow \downarrow \downarrow\rangle_{1423}-e^{-i \gamma}|\downarrow \downarrow \uparrow \uparrow\rangle_{1423}\right) .
\end{aligned}
$$

Here $A$ represents " 14 " and $B$ represents " 23 ”, $\gamma=\left(\varphi_{1}+\varphi_{4}-\varphi_{2}-\right.$ $\left.\varphi_{3}\right) / 2$, and $\varphi_{A}=\varphi_{1}-\varphi_{4}, \varphi_{B}=\varphi_{2}-\varphi_{3}$ are relative AC phases for meeting locations $\mathrm{A}$ and $\mathrm{B}$ acquired by four particles moving along different paths. It is worth to mention that AC effect usually concerns a single particle moving around a line charge, however here none of the moving paths of four particles encircles the line charge, though the combination of four corresponding paths actually makes a circle.

Next we perform local projective measurements on two particle pairs $(1,4)$ and $(2,3)$ along arbitrary directions $\vec{n}_{A}=\left(\sin \xi_{A} \cos \theta_{A}\right.$, $\left.\sin \xi_{A} \sin \theta_{A}, \cos \xi_{A}\right)$ and $\vec{n}_{B}=\left(\sin \xi_{B} \cos \theta_{B}, \sin \xi_{B} \sin \theta_{B}\right.$, $\left.\cos \xi_{B}\right)$, respectively. The projectors are defined as $\hat{\mathcal{P}}(i, j)=$ $\left|\bar{i}_{n_{A}} \bar{j}_{n_{B}}\right\rangle\left\langle\bar{i}_{n_{A}} \bar{j}_{n_{B}}\right|,(i, j=0,1)$, where

$$
\begin{aligned}
& \left|\overline{0}_{\vec{n}}\right\rangle=(|+\vec{n},-\vec{n}\rangle-|-\vec{n},+\vec{n}\rangle) / \sqrt{2}, \\
& \left|\overline{1}_{\vec{n}}\right\rangle=(|+\vec{n},-\vec{n}\rangle+|-\vec{n},+\vec{n}\rangle) / \sqrt{2},
\end{aligned}
$$

which are respectively the singlet state and the triplet state with $M=$ 0 written in terms of the following states: $|+\vec{n}\rangle=\cos \frac{\xi}{2}|\uparrow\rangle+$ $\sin \frac{\xi}{2} e^{i \theta}|\downarrow\rangle,|-\vec{n}\rangle=\sin \frac{\xi}{2}|\uparrow\rangle-\cos \frac{\xi}{2} e^{i \theta}|\downarrow\rangle$. Here we choose the vectors $\vec{n}_{A}$ and $\vec{n}_{B}$ in the $x y$-plane, i.e., $\xi_{A}=\xi_{B}=\pi / 2$. Let us denote $P(i, j)=\left\langle\Psi_{\mathrm{f}}|\hat{\mathcal{P}}(i, j)| \Psi_{\mathrm{f}}\right\rangle$ as the joint probabilities satisfying the nosignaling condition, and based on which the correlation function is defined as

$$
C_{A B}=\frac{\sum_{i, j=0,1}(-1)^{i+j} P(i, j)}{\sum_{i, j=0,1} P(i, j)} .
$$

After some calculations, we obtain the explicit result of the correlation function as

$$
C_{A B}(\vec{a}, \vec{b})=-\vec{a} \cdot \vec{b}
$$

where $\vec{a}=\left(\sin \theta_{A} \cos \varphi_{A}, \sin \theta_{A} \sin \varphi_{A}, \cos \theta_{A}\right)$ and $\vec{b}=\left(\sin \theta_{B} \cos \varphi_{B}\right.$, $\left.\sin \theta_{B} \sin \varphi_{B}, \cos \theta_{B}\right)$ are two unit three-dimensional vectors. Here the vectors $\vec{a}$ and $\vec{b}$ (or say $\varphi_{A}, \varphi_{B}, \theta_{A}, \theta_{B}$ ) are experimentally controllable: The parameters $\varphi_{A}, \varphi_{B}$ (i.e., $\left.\varphi_{i}, i=1,2,3,4\right)$ are the relative AC phase shifts of the four particles determined by the locations $A, B$ and the paths $\ell_{i}$; and the parameters $\theta_{A}, \theta_{B}$ come from the selection of directions in the projective measurements for each particle pair at $A$ and $B$. Actually, the correlation function (15) is equivalent to $C_{A B}(\vec{a}, \vec{b})=\left\langle\Psi_{\mathrm{i}}|\vec{\Sigma} \cdot \vec{a} \otimes \vec{\Sigma} \cdot \vec{b}| \Psi_{\mathrm{i}}\right\rangle$, which is just similar to that of two usual qubits under the joint measurement $\vec{\sigma} \cdot \vec{a} \otimes \vec{\sigma} \cdot \vec{b}$ on the singlet state. This correspondence also provides a reasonable explanation on why the AC effect can be used to test both the Bell-Clauser-HorneShimony-Holt (Bell-CHSH) inequality ${ }^{2}$ in Ref. 17 and the Leggett inequality in this work.

Reference 17 proposed to test the Bell-CHSH inequality

$$
\left|C(\vec{a}, \vec{b})+C\left(\vec{a}^{\prime}, \vec{b}\right)+C\left(\vec{a}, \vec{b}^{\prime}\right)-C\left(\vec{a}^{\prime}, \vec{b}^{\prime}\right)\right| \leq 2
$$

using the AC effect. There are four measurement settings in the inequality (16), i.e., $\vec{a}, \vec{b}, \vec{a}^{\prime}, \vec{b}^{\prime}$. To attain maximal violation of the inequality, it is sufficient to put the four measurement settings in the same plane, i.e., one may always choose $\theta_{A}=\theta_{A^{\prime}}=\theta_{B}=\theta_{B^{\prime}}=\pi / 2$ if the Bell-CHSH inequality is tested. By properly selecting two locations $A, A^{\prime}$ for Alice where particle pair $(1,4)$ meets, and two locations $B, B^{\prime}$ for Bob where particle pair $(2,3)$ meets, and adjusting the phase shifts as $\varphi_{A}=0, \varphi_{A^{\prime}}=\pi / 2, \varphi_{B}=\pi / 4, \varphi_{B^{\prime}}=-\pi / 4$, or say $\vec{a}=(1,0,0)$,

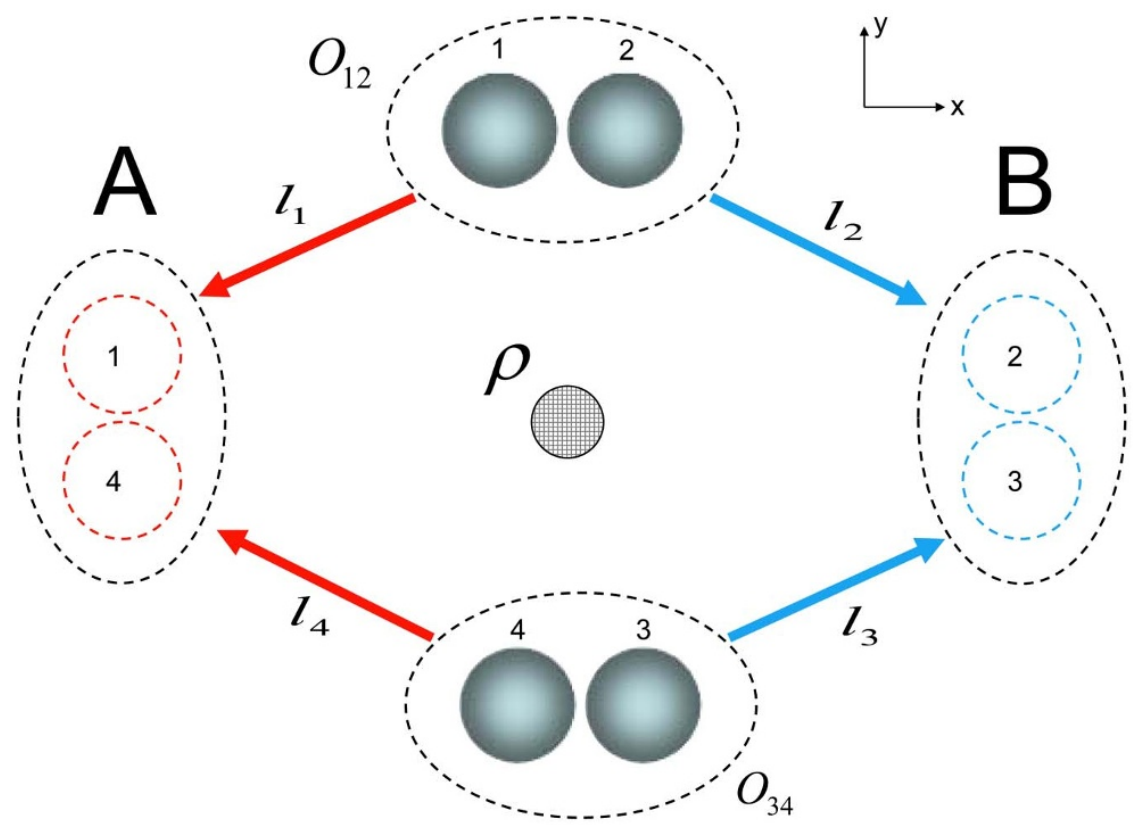

Figure $1 \mid$ A schematic illustration of experiment proposal. We let the two sources be located at points $O_{12}$ and $O_{34}$ on the $x y$-plane respectively, and invoke an impenetrable line charge (with charge density $\rho$ ) oriented along the $z$-axis. After the four particles are emitted from the two sources, we then move particle 1 from location $O_{12}$ to location $A$ along path $\ell_{1}$, and move particle 4 from location $O_{34}$ to meet particle 1 at location $A$ along path $\ell_{4}$. The motion of the particles are influenced by the electric field of line charge as shown in Eq. (5) and accordingly the corresponding AC phase shifts are $\varphi_{1}$ and $\varphi_{4}$ for particles 1 and 4 respectively. Similarly, we move particle 2 from location $O_{12}$ to location $B$ along path $\ell_{2}$, and move particle 3 from location $O_{34}$ to meet particle 2 at location $B$ along path $\ell_{3}$, and the corresponding AC phase shifts are $\varphi_{2}$ and $\varphi_{3}$ for particles 2 and 3 respectively. 


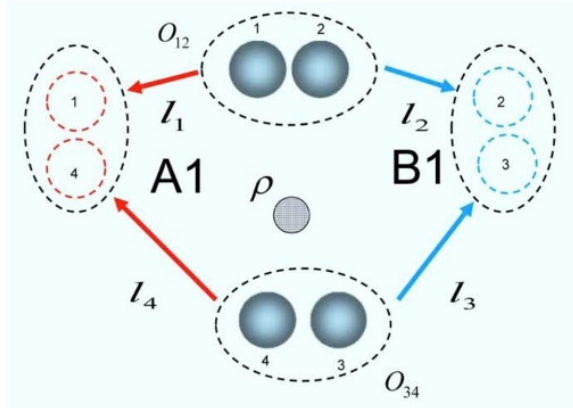

(a)

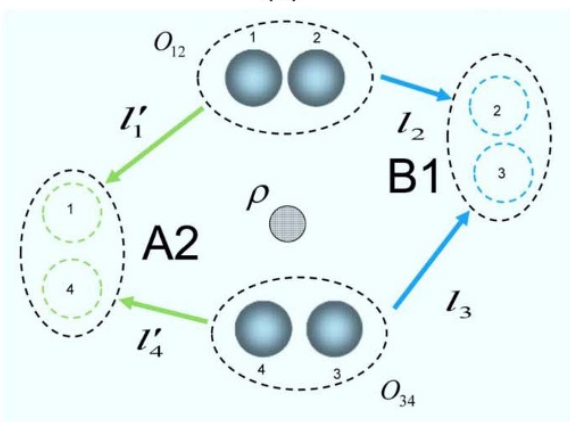

(c)

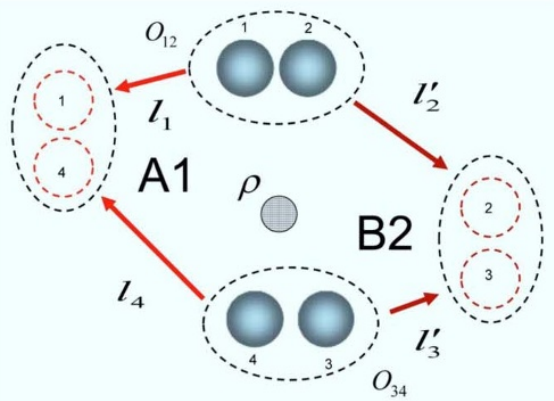

(b)

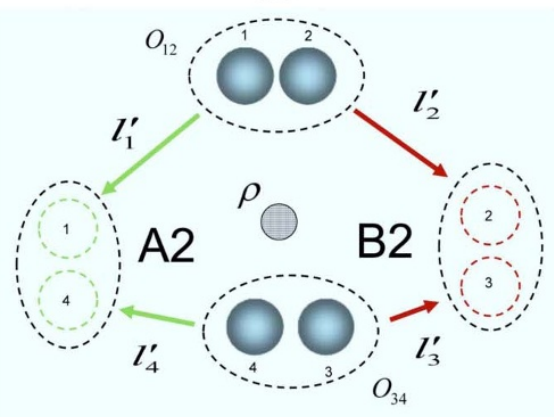

(d)

Figure $2 \mid$ Illustration of different locations and trajectories in space. Properly choose three locations $A_{i}(i=1,2,3)$ for Alice where particle pair $(1,4)$ meets, and six locations $B_{i} / B_{i}^{\prime}(i=1,2,3)$ for Bob where particle pair $(2,3)$ meets, and control the different paths such that we arrive at the experimental settings given in Ref. 14. (a) Illustration of locations $A_{1}$ and $B_{1}$, and paths $\ell_{1}, \ell_{2}, \ell_{3}, \ell_{4}$; (b) Illustration of 1 ocations $A_{1}$ and $B_{2}$, and paths $\ell_{1}$, $\ell_{2}^{\prime}, \ell_{3}^{\prime}, \ell_{4}$; (c) Illustration of locations $A_{2}$ and $B_{1}$, and paths $\ell_{1}^{\prime}, \ell_{2}, \ell_{3}, \ell_{4}^{\prime}$; (d) Illustration of locations $A_{2}$ and $B_{2}$, and paths $\ell_{1}^{\prime}, \ell_{2}^{\prime}, \ell_{3}^{\prime}, \ell_{4}^{\prime}$. Other locations and their corresponding paths can be given in a similar way.

$\vec{a}^{\prime}=(0,1,0), \vec{b}=(1 / \sqrt{2}, 1 / \sqrt{2}, 0)$, and $\vec{b}^{\prime}=(1 / \sqrt{2},-1 / \sqrt{2}, 0)$, then the right-hand side of (16) achieves $2 \sqrt{2}$ and thus the Bell-CHSH inequality is maximally violated. The violation of the Bell inequality rules out local realistic theories from quantum mechanics.

To test Leggett's inequality (3), we need totally nine measurement settings, i.e., $\vec{a}_{1}, \vec{a}_{2}, \vec{a}_{3}, \vec{b}_{1}, \vec{b}_{1}^{\prime}, \vec{b}_{2}, \vec{b}_{2}^{\prime}, \vec{b}_{3}, \vec{b}_{3}^{\prime}$. Since $\vec{e}_{i}(i=1,2,3)$ is an orthogonal basis, the nine measurement settings cannot lie in the same plane. Properly select three locations $A_{i}(i=1,2,3)$ for Alice where particle pair $(1,4)$ meets, and six locations $B_{i} / B_{i}^{\prime}(i=1,2,3)$ for Bob where particle pair $(2,3)$ meets (see Fig. 2 ), and adjust the nine different paths and nine directions of the projectors such that the measurement settings are $\left(\theta_{A_{1}}, \varphi_{A_{1}}\right)=(\pi / 2,0),\left(\theta_{A_{2}}, \varphi_{A_{2}}\right)=$ $(\pi / 2, \pi / 2),\left(\theta_{A_{3}}, \varphi_{A_{3}}\right)=(0,0),\left(\theta_{B_{1}}, \varphi_{B_{1}}\right)=(\pi / 2, \varphi / 2),\left(\theta_{B_{1}^{\prime}}, \varphi_{B_{1}^{\prime}}\right)=$ $(\pi / 2,-\varphi / 2), \quad\left(\theta_{B_{2}}, \varphi_{B_{2}}\right)=(\pi / 2-\varphi / 2, \pi / 2), \quad\left(\theta_{B^{\prime}}, \varphi_{B_{2}^{\prime}}\right)=(\pi / 2+$ $\varphi / 2, \pi / 2),\left(\theta_{B_{3}}, \varphi_{B_{3}}\right)=(\varphi / 2,0),\left(\theta_{B_{3}^{\prime}}, \varphi_{B_{3}^{\prime}}\right)=(\varphi / 2, \pi)$, we arrive at the experimental settings given in Ref. 14. Based on which the six correlation functions in Eq. (3) are all equal to $-\cos (\varphi / 2)$, and consequently for $|\varphi| \in\left(0,4 \tan ^{-1}\left(\frac{1}{3}\right)\right)$, the Leggett inequality (4) is violated. The violation of the Leggett inequality implies that nonlocal realistic theories are not compatible with quantum mechanics. In the $\mathrm{AC}$ experiment, the invalidity of both the Bell inequality and the Leggett inequality suggests that quantum mechanics is neither local nor realistic. The result is consistent with the works in the literatures ${ }^{1-14}$ based on the experiment of entangled photons.

\section{Discussion}

Let us make some discussion on the possible implementation of our scheme in physical systems. One possible system to explore the our scheme experimentally is a calcium atomic polarization interferometer as investigated in Ref. 19. Encode two magnetic substates of the excited state $\left|{ }^{3} P_{1}\right\rangle$ as computational basis, $|\uparrow\rangle \equiv \mid{ }^{3} P_{1},+$ $1\rangle$ and $|\downarrow\rangle \equiv\left|{ }^{3} P_{1},-1\right\rangle$, the phase difference between $|\uparrow\rangle$ and $|\downarrow\rangle$ accumulated during the evolution includes two parts, one is dynamical phase and the other one is nothing but the AC phase. As we know, the presence of dynamical phase may destroy the potential robustness of our scheme since it is sensitive to noise. Fortunately the dynamical part can be canceled out via interferometer, as shown in Ref. 19, and therefore one only has the AC phase in the experiment. Due to the topological property of the AC phase, the experiment offers a promising fault-tolerant method to test Leggett's inequality. The experimental achievement in the literature ${ }^{19}$ tells us that our test of Leggett's inequality using the AC effect is possibly realizable with current techniques in an experiment of a calcium atomic polarization interferometer.

In summary, we have proposed a scheme to test the two-qubit Leggett inequality using two entangled spin-1/2 particle pairs emitted from two sources in the presence of a line charge. Pseudo-Pauli matrices are introduced such that these four particles can be viewed as a total "two-qubit" system. The influence of the AC effect on each entangled spin pair is found to be equivalent to a rotation in terms of the pseudo-Pauli operators. Based on the final state of the physical system, two-qubit-type correlation functions with controllable parameters can be calculated from joint probabilities for the measurement of the two particle pairs with $M=0$. The Leggett inequality is found to be violated, which implies the invalidity of nonlocal realistic theories. The merit of our scheme lies at robustness against local inaccuracy, and thus our scheme of testing the Leggett inequality is tolerant to some local inaccuracies. As is well known, photon-based experiments often encounter loophole problems, such as errors in the detectors and detecting systems. The existence of loopholes may affect the validity of the experiments, and hence the investigation of 
loophole-free experiments is a good alternative. This makes our scheme totally different from the known experiments on testing the Leggett inequality in the literatures ${ }^{11-14}$.

1. Bell, J. S. On The Einstein Podolsky Rosen Paradox. Physics (Long Island City, N. Y.) 1, 195 (1964).

2. Clauser, J., Horne, M., Shimony, A. \& Holt, R. Proposed experiment to test local hidden-variable theories. Phys. Rev. Lett. 23, 880 (1969).

3. Freedman, S. J. \& Clauser, J. F. Experimental test of local hidden-variable theories. Phys. Rev. Lett. 28, 938 (1972).

4. Aspect, A., Dalibard, J. \& Roger, G. Experimental Test of Bell's Inequalities Using Time- Varying Analyzers. Phys. Rev. Lett. 49, 1804 (1982).

5. Weihs, G., Jennewein, T., Simon, C., Weinfurter, H. \& Zeilinger, A. Violation of Bell's inequality under strict Einstein locality conditions. Phys. Rev. Lett. 81, 5039 (1998).

6. Rowe, M. A. et al. Experimental violation of a Bell's inequality with efficient detection. Nature 409, 791 (2001).

7. Zeilinger, A. Testing Bell's inequalities with periodic switching. Phys. Lett. A 118, 1 (1986)

8. Aspect, A. Bell's inequality test: more ideal than ever. Nature 398, 189 (1999).

9. Grangier, P. Quantum physics: Count them all. Nature 409, 774 (2001).

10. Leggett, A. J. Nonlocal hidden-variable theories and quantum mechanics: An incompatibility theorem. Found. Phys. 33, 1469 (2003).

11. Gröblacher, S. et al. An experimental test of non-local realism. Nature 446, 871 (2007).

12. Paterek, T. et al. Experimental test of nonlocal realistic theories without the rotational symmetry assumption. Phys. Rev. Lett. 99, 210406 (2007).

13. Branciard, C. et al. Experimental falsification of Leggett's nonlocal variable model. Phys. Rev. Lett. 99, 210407 (2007).

14. Branciard, C. et al. Testing quantum correlations versus single-particle properties within Leggett's model and beyond. Nature Phys. 4, 681 (2008).

15. Aharonov, Y. \& Bohm, D. Significance of electromagnetic potentials in the quantum theory. Phys. Rev. 115, 485 (1959).
16. Aharonov, Y. \& Casher, A. Topological quantum effects for neutral particles. Phys. Rev. Lett. 53, 319 (1984).

17. Pati, A. K. Testing Bell's inequality using the Aharonov-Casher effect. Phys. Rev. A 58, R1 (1998).

18. Hacyan, S. Bell's inequality violation with four entangled fermions. Phys. Rev. A 56, R2489 (1997)

19. Yanagimachi, S., Kajiro, M., Machiya, M. \& Morinaga, A. Direct measurement of the Aharonov-Casher phase and tensor Stark polarizability using a calcium atomic polarization interferometer. Phys. Rev. A 65, 042104 (2002).

\section{Acknowledgements}

We thank Prof A. J. Leggett for very helpful suggestions and valuable comments. J.L.C. is supported by National Basic Research Program (973 Program) of China under Grant No. 2012CB921900, NSF of China (Grant Nos. 10975075 and 11175089). This work is partly supported by National Research Foundation and Ministry of Education of Singapore (Grant No. WBS: R-710-000-008-271).

\section{Author contributions}

J.L.C. initiated the idea. J.L.C., H.Y.S. and C.W. derived the results. J.L.C., H.Y.S., C.W., D.L.D. and C.H.O. wrote the main manuscript text. H.Y.S. prepared the figure. All authors reviewed the manuscript.

\section{Additional information}

Competing financial interests: The authors declare no competing financial interests.

How to cite this article: Su, H.-Y., Chen, J.-L., Wu, C.F., Deng, D.-L. \& Oh, C.H. Testing Leggett's Inequality Using Aharonov-Casher Effect. Sci. Rep. 3, 2492; DOI:10.1038/ srep02492 (2013).

\footnotetext{
(c) (i) (2) This work is licensed under a Creative Commons AttributionNonCommercial-ShareAlike 3.0 Unported license. To view a copy of this license, visit http://creativecommons.org/licenses/by-nc-sa/3.0
} 